\title{
High density lipoprotein and kidney dysfunction among post kidney transplant patients
}

\author{
Laiq $S^{1}$, Al Salmi I ${ }^{*}$ and Hannawi $S^{2}$ \\ ${ }^{1}$ The Renal Medicine Department, the Royal Hospital, Oman \\ ${ }^{2}$ The Medical Department, MOHAP, Dubai, UAE
}

\begin{abstract}
Introduction: Dyslipidemia is commonly found in patients after kidney transplantation and it is associated with cardiovascular complications. Kidney transplant patients suffer high mortality due to cardiovascular diseases.

Objective: Patients who have undergone kidney transplant, and present with chronic kidney disease (CKD) often have poor high-density lipoprotein (HDL) control. Hence, this study aims to establish a correlation between serum HDL levels in transplant recipients with kidney function.

Design: A retrospective cross-sectional study, which examined 240 patients who underwent kidney transplant, and were followed up between 2006 and 2012 . HDL values along estimated glomerular filtration rate (eGFR) were recorded, on a yearly basis, using Epi- Data entry software and then transferred to SPSS software for analysis.

Results: A total of 106 patients are included out of our initial sample of 240 , the rest 134 were excluded, for various causes. the age ranged from 14 to 76 years, with a mean of 42.7 years, and a median of 42 years. 62 of the included participants were males, 44 were female. Year of transplant varied from 1981 to 2013.32 of the patients underwent live related renal transplant and 78 underwent commercial transplant. From the analysis we interpreted, with a continuity correction of $\mathrm{p}=1.000$, that there was no statistically significant association between the two categorical variables $(\mathrm{p}>0.05)$.

Conclusion: We found no statistically significant association between worsening HDL levels and renal function decline in our study population of patients who underwent renal transplant.
\end{abstract}

\section{Introduction}

There is a progressive rise in the number of kidney transplant patients world-wide and in Oman [1,2]. A significant number of them whether transplanted from a living relative or commercial unrelated kidney transplant, eventually develop kidney dysfunction and failure of the graft kidney for various reasons. Patients with kidney transplantation are at increased risk of accelerated atherosclerosis and a marked increase in cardiovascular (CV) mortality [3]. Several factors are involved in the pathogenesis of atherosclerosis and CV disease in chronic kidney disease (CKD). Post-renal transplant (Tx) patients exhibit a high CV morbidity and mortality due to accumulation of $\mathrm{CV}$ risk factors such as oxidative stress, inflammation, hypertension, endothelial dysfunction, vascular calcification, medications, metabolic abnormalities and dyslipidemia [4]. Some of the transplant kidney dysfunctions and failures have causes that are easily identified, including infections, drugs, recurrence of original kidney disease [5]. Other transplant failures are multifactorial, and a specific cause cannot usually be identified [6].

It is not surprising that in the general population, increasing HDL cholesterol concentrations are associated with decreased atherosclerosis burden and reduced CV mortality [7]. There are few studies in the literature pertaining to correlating HDL with renal dysfunction in post renal transplant patients [8-10], however none have shown a clear association or disproved one with strong evidence, hence this topic remains controversial, also no such study has been done in our citizens in the Sultanate. Therefore, this study examines the correlation of lipids most specifically HDL (High density lipoprotein) levels in kidney transplant patients in whom a specific cause of kidney dysfunction was not very well identified, and those who suffered a gradual decline in renal function due to unknown causes.

\section{Aim}

The aim of this study is to evaluate the relationship between HDL serum level in transplant recipients with subsequent kidney dysfunction. Further analysing if differences occur between live related and commercial kidney transplants. Our hypothesis is that poorly controlled HDL levels may predispose the transplanted kidney graft to kidney dysfunction and ultimate end stage kidney disease in patients that received kidney transplant either from live related or commercial transplantation, in those who no other specific cause was isolated.

\section{Methodology}

This is a cross sectional design study of all patients that were seen at the Renal Medicine Department, Royal Hospital, Muscat. All relevant information about the patient was collected and entered into Epi- Data entry software and then transferred to SPSS software for analysis. The

${ }^{*}$ Correspondence to: Issa Al Salmi, The Renal Medicine Department, The Royal Hospital, Oman, Tel: 96892709000; Fax: 96824599966; E-mail: isa@ausdoctors.net

Received: September 16, 2018; Accepted: September 26, 2018; Published: September 28, 2018 
collected data included demographic data, lipids data, renal function data, liver function data, metabolic data, inflammatory laboratory data and urinalysis data.

There are a total of 1213 patients registered as kidney transplant recipients at Royal hospital as of January 2017 when the data was collected. As this is a across section study, we took a sample population of 240 patients, randomized, to optimally represent the target population. The study period was 2006-2016. The data was collected from each patient using the Shifa medical system, yearly at the last follow up of each patient every year. Each patient's history was closely studied from the available detailed medical records of the computerized data system.

Patients that lost follow up for over a year, or those that had less than 3 years of follow up were excluded from the study. Patient's that had an identifiable cause confirmed for renal function decline, were further excluded from analysis.

\section{Exclusion criteria}

Patients that lost follow up for over a year, or those that had less than 3 years of follow up were excluded from the study. Patient's that had an identifiable cause confirmed for renal function decline, including immune mediated rejections (most commonly), obstructive uropathy, recurrence of native renal pathologies, graft infections, were further excluded from analysis. This resulted in an exclusion of 134 patients.

\section{Inclusion criteria}

The remaining 106 patients in our sample size were included, for whom a clear cause for worsening renal failure was either not clear from the notes, labs and/or histopathology. A further description of this subgroup is further elucidated in the results

Parameters collected were age, sex, nationality, year and country of renal transplant, and whether it was living related donor or an unrelated donor (commercial). HDL values along with corresponding renal function values (eGFR or estimated glomerular filtration rate) were recorded. As our study is focused on HDL values and its possible correlation with graft function, other lipid parameters were not analysed currently.

Parameters collected were age, sex, nationality, year and country of renal transplant, and whether it was living related donor or an unrelated donor (commercial). HDL values along with corresponding renal function values (eGFR or estimated glomerular filtration rate) were recorded.

A renal function decline was defined as a reduction of $>5 \mathrm{~mL} /$ $\mathrm{min} / 1.73 \mathrm{~m}^{2}$, from patient's baseline eGFR value post renal transplant. Any decrease in baseline HDL value more than $0.2 \mathrm{mmol} / \mathrm{L}$, during the patient's follow up was considered as worsening in the HDL parameter.

\section{Results}

Table 1 shows the descriptive statistics as outlined in cross tabulation for our studied population. A total of 134 patients were excluded, where a clear cause explaining their kidney function decline was reported. The most commonly identified cause was immune mediated graft rejection, with other causes being infective, and recurrence of native kidney disease in that order. Some others were excluded either due to insufficient data, missing data, or lack of adequate follow up (follow up less than 3 years).

Thus, in our final sample of patient's that were studied after applying the exclusion criteria, the age ranged from 14 to 76 years, with a mean of 42.7, and a median of 42 years. 62 of the included study participants were males, 44 were female. Year of transplant varied from 1981 to 2013.32 of the patients underwent live related renal transplant and 78 underwent commercial transplant.

Table 1 describes our study population. In which a total of 106 patients are included out of our initial sample of 240, the rest 134 were excluded, for causes outlined above.

A total of 46 of the patients who did not have any measurable change in HDL values, did not have any decline in kidney function, 40 others had a measurable decline in kidney function, but no significant reduction in their overall baseline HDL values.

10 of the patients, who did have a measurable change in their HDL values, did not have any decline in kidney function. 10 of them had a measurable decline in kidney function, with significant reduction in their overall baseline HDL values. These last 10 patients would be in accordance with our hypothesis.

The statistical analysis of the above data is detailed in the Table 2.

From table 2, we can interpret, with a continuity correction of $p=1.000$, that there was NO statistically significant association between the two categorical variables ( $\mathrm{p}>0.05)$.

\section{Discussion}

The present study showed that poorly controlled HDL levels did not seem to predispose the transplanted kidney graft to dysfunction in our patients, in those whom we could not identify a clear reason for transplant dysfunction or failure. The study has importance from a public health prospective, as to whether intervention is needed on a large scale in this particular subset of patient with low or worsening HDL level post renal transplant. Also, it has cost implications for patients, especially those who endured a lot to get an organ transplant and may face loss of its function or eventual failure. Therefore, in clinical practice, the focus should be on dyslipidemic patients mainly on total cholesterol and LDL (low density lipoprotein) [11,12].

Table 1. HDL/RFT * Renal Fx Decline Crosstabulation

\begin{tabular}{|c|c|c|c|c|c|}
\hline \multirow{2}{*}{\multicolumn{3}{|c|}{ HDL/RFT }} & \multicolumn{2}{|c|}{ Renal Fx Decline } & \multirow{3}{*}{\begin{tabular}{|l|} 
Total \\
86 \\
\end{tabular}} \\
\hline & & & \multirow{2}{*}{\begin{tabular}{|l|} 
No \\
46 \\
\end{tabular}} & \multirow{2}{*}{\begin{tabular}{|l} 
Yes \\
40 \\
\end{tabular}} & \\
\hline \multirow{6}{*}{ HDL decrease with RFT } & \multirow{3}{*}{ No change in HDL } & Count & & & \\
\hline & & $\%$ within HDL dec w RFT & $53.50 \%$ & $46.50 \%$ & $100.00 \%$ \\
\hline & & $\%$ within Renal Fx decline & $82.10 \%$ & $80.00 \%$ & $81.10 \%$ \\
\hline & \multirow{3}{*}{ HDL worsened } & Count & 10 & 10 & 20 \\
\hline & & $\%$ within HDL dec w RFT & $50.00 \%$ & $50.00 \%$ & $100.00 \%$ \\
\hline & & $\%$ within Renal Fx decline & $17.90 \%$ & $20.00 \%$ & $18.90 \%$ \\
\hline \multirow{3}{*}{\multicolumn{2}{|c|}{ Total }} & Count & 56 & 50 & 106 \\
\hline & & $\%$ within HDL dec w RFT & $52.80 \%$ & $47.20 \%$ & $100.00 \%$ \\
\hline & & $\%$ within Renal Fx decline & $100.00 \%$ & $100.00 \%$ & $100.00 \%$ \\
\hline
\end{tabular}


Table 2. Chi-Square Tests

\begin{tabular}{|c|c|c|c|c|c|}
\hline & Value & df & $\begin{array}{l}\text { Asymp. Sig. } \\
\text { (2-sided) }\end{array}$ & $\begin{array}{l}\text { Exact Sig. } \\
\text { (2-sided) }\end{array}$ & $\begin{array}{l}\text { Exact Sig. } \\
\text { (1-sided) }\end{array}$ \\
\hline Pearson Chi-Square & $0.040^{\mathrm{a}}$ & 1 & 0.842 & - & - \\
\hline Continuity Correction ${ }^{\mathrm{b}}$ & 0.000 & 1 & 1.000 & - & - \\
\hline Likelihood Ratio & 0.040 & 1 & 0.842 & - & - \\
\hline Fisher's Exact Test & - & - & - & 1.000 & 0.559 \\
\hline Linear-by-Linear Association & 0.039 & 1 & 0.844 & - & - \\
\hline $\mathrm{N}$ of Valid Cases & 53 & - & - & - & - \\
\hline
\end{tabular}

This cross-sectional design study showed no statistically significant association between HDL level and eGFR level among patients with 3 years post kidney transplant with no apparent cause for the decrease in kidney function. However, there were previous studies that have been done to study correlation of HDL with chronic kidney disease (CKD), in non-transplanted patients [13]. Some even provide evidence to suggest low levels of HDL cause reno-vascular atherosclerosis, glomerulosclerosis, and may even lead to tubulo-interstitial injury [13-17]. Another study showed highest decline in renal function in dyslipidemic patients with the lowest HDL levels [18].

In the present study, we used systematic random samples to collect data, where the values of the estimated statistic neither consistently overestimate nor consistently underestimate the value of the population parameters. Also, Randomization eliminates personal choice and hence eliminates the possibility of subjective selection bias. In addition, the randomization distribution provides a basis for statistical inference. Despite keeping a low threshold for HDL changes of $0.2 \mathrm{mmol} / \mathrm{L}$ we were unable to obtain a statistically significant correlation between worsening of HDL levels and renal function decline. Further analysis in a study suggested that the relationship between $\mathrm{HDL}$ and $\mathrm{CV}$ outcomes may not be causal, and that HDL may only be a marker for renal outcomes [13], with the additional risk factors in our post renal transplant patients, with their own associated risk factors [19] and medication side effects including high dose steroids and cyclosporin toxicity $[19,20]$, further confounds our analysis to single out HDL as a correlation or an association, in post kidney transplant dysfunction or failure.

Dyslipidaemia is commonly found in patients after kidney transplantation and it is associated with CV complications. Kidney transplant patients suffer high mortality due to CV diseases [21,22]. Some of the side effects may be dose related [21,22]. CNIs have a narrow therapeutic window. Hence, monitoring their levels is important for their administration [23]. Low level of the HDL2 subfraction has been reported after kidney transplantation [24]. In the present study, the available data does not allow for differentiation in HDL subclasses, especially HDL2 which is known to be associated improved CV outcomes [24], and thus possibly better kidney function outcomes. A study investigated the relationships between doses and blood levels of calcineurin inhibitors (CNIs) [cyclosporin A (CsA) and tacrolimus (Tac)] and lipid parameters and found that there was no significant difference in TG and HDL-CHOL plasma levels [25].

However, there are previous studies done studying correlation of dyslipidaemias with renal function decline, albeit in patients who did not undergo renal transplantation showing inconsistent results $[15,26-$ 28]. One such study did a post ad-hoc analysis and found "treatment with fibrates does not seem to confer renoprotection", fibrates here being a drug to improve HDL levels [15]. The study further states that "most studies that have evaluated the effects of dyslipidaemia on renal disease have been performed with patients with pre-existing renal disease or other risk factors for renal disease, such as hypertension and diabetes [15].

A study by Yau, et al. found no associations between kidney dysfunction and cyclosporine levels in whole blood, total plasma, or lipoprotein fractions among post bone marrow transplant patients [29]. The ratios of maximum and mean high-density low-density lipoprotein cyclosporine concentrations were greatest in patients with kidney dysfunction ( $p$ less than 0.001) [29]. They concluded that therapeutic drug monitoring of cyclosporine in various biologic fluids does not predict onset of drug-associated kidney dysfunction [29]. However, the relative role of high-density to low-density lipoprotein transport of cyclosporine may provide an index of renal functional changes associated with the agent [29].

While our randomization minimizes bias (both unbiased and efficient), however, the present study sample size was small, and being confined to data only obtained from Royal hospital follow up patients in Oman. A broader study which includes all patients including other health institutions in Oman, would provide a clearer picture in correlating HDL levels with post renal transplant dysfunction.

Also, even though we did try to exclude patients with clearly apparent causes of renal dysfunction from the analysis to reduce error, there remains the possibility that poor HDL control may have contributed to their adverse outcome, and conversely those that were included may have another unknown cause of their kidney dysfunction, and in them HDL served merely as a marker.

\section{Conclusion}

The present study found no statistically significant association between worsening HDL levels and kidney function decline in patients who were followed up routinely for kidney transplant in the nephrology out patient's department. In view of cost implications for our patients, therefore, in clinical practice, the focus should be on dyslipidemic patients mainly on total cholesterol and LDL (low density lipoprotein). This importance from a public health prospective, as to whether intervention is needed on a large scale in this particular subset of patient post renal transplant.

\section{References}

1. Al Alawi I, Al Salmi I, Al Mawali A, Al Maimani Y, Sayer JA (2017) End-Stage Kidney Failure in Oman: An Analysis of Registry Data with an Emphasis on Congenital and Inherited Renal Diseases. Int J Nephrol 2017: 1-7. [Crossref]

2. Al Ismaili F, Al Salmi I, Al Maimani Y, Metry AM, Al Marhoobi H, et al. (2016) Epidemiological Transition of End-Stage Kidney Disease in Oman. Kidney Int Rep 2: 27-35. [Crossref]

3. Meier-Kriesche HU, Schold JD, Srinivas TR, Reed A, Kaplan B (2004) Kidney transplantation halts cardiovascular disease progression in patients with end-stage renal disease. Am J Transplant 4: 1662-1668.

4. London GM (2005) Vascular disease and atherosclerosis in uremia. Nefrologia 25 91-95. [Crossref]

5. Marcen R (2009) Immunosuppressive drugs in kidney transplantation: impact on patient survival, and incidence of cardiovascular disease, malignancy and infection. Drugs 69: 2227-2243.

6. Sellares J, de Freitas DG, Mengel M, Reeve J, Einecke G, et al. (2011) Understanding the causes of kidney transplant failure: the dominant role of antibody-mediated rejection and nonadherence. Am J Transplant 12: 388-399.

7. Ravnskov U, Diamond DM, Hama R, Hamazaki T, Hammarskjold B, et al. (2016) Lack of an association or an inverse association between low-density-lipoprotein cholesterol and mortality in the elderly: a systematic review. BMJ 6: e010401. 
8. Kimak E, Bylina J, Solski J, Halabis M, Baranowicz-Gaszczyk I, et al. (2013) Association between lipids, lipoproteins composition of HDL particles and triglyceriderich lipoproteins, and LCAT and CETP activity in post-renal transplant patients. Cell Biochem Biophys 67: 695-702

9. Kimak E, Halabis M, Baranowicz-Gaszczyk I (2010) Relationships between serum lipid, lipoprotein, triglyceride-rich lipoprotein, and high-density lipoprotein particle concentrations in post-renal transplant patients. J Zhejiang Univ Sci B 11: 249-257.

10. Kimak E, Solski J, Baranowicz-Gaszczyk I, Ksiazek A (2006) A long-term study of dyslipidemia and dyslipoproteinemia in stable post-renal transplant patients. Ren Fail 28: 483-486.

11. Agarwal A, Prasad GV (2016) Post-transplant dyslipidemia: Mechanisms, diagnosis and management. World J Transplant 6: 125-134.

12. Cattran DC, Steiner G, Wilson DR, Fenton SA (1979) Hyperlipidemia after renal transplantation: natural history and pathophysiology. Ann Intern Med 91: 554-559.

13. Bowe B, Xie Y, Xian H, Balasubramanian S, Al-Aly Z (2016) Low levels of highdensity lipoprotein cholesterol increase the risk of incident kidney disease and its progression. Kidney Int 89: 886-896. [Crossref]

14. Ganda A, Magnusson M, Yvan-Charvet L, Hedblad B, Engstrom G, et al. (2013) Mild renal dysfunction and metabolites tied to low HDL cholesterol are associated with monocytosis and atherosclerosis. Circulation 127: 988-996.

15. Cases A, Coll E (2005) Dyslipidemia and the progression of renal disease in chronic renal failure patients. Kidney Int Suppl 68: 87-93. [Crossref]

16. Morton J, Zoungas S, Li Q, Patel AA, Chalmers J, et al. (2012) Low HDL cholestero and the risk of diabetic nephropathy and retinopathy: results of the ADVANCE study. Diabetes Care 35: 2201-2206.

17. Baragetti A, Norata GD, Sarcina C, Rastelli F, Grigore L, et al. (2013) High density lipoprotein cholesterol levels are an independent predictor of the progression of chronic kidney disease. J Intern Med 274: 252-262.

18. Mänttäri M, Tiula E, Alikoski T, Manninen V (1995) Effects of hypertension and dyslipidemia on the decline in renal function. Hypertension 26: 670-675. [Crossref]
19. Ojo AO, Hanson JA, Wolfe RA, Leichtman AB, Agodoa LY, et al. (2000) Long-term survival in renal transplant recipients with graft function. Kidney Int 57: 307-313. [Crossref]

20. Veenstra DL, Best JH, Hornberger J, Sullivan SD, Hricik DE (1999) Incidence and long-term cost of steroid-related side effects after renal transplantation. Am J Kidney Dis 33: 829-839. [Crossref]

21. Ligtenberg G, Hené RJ, Blankestijn PJ, Koomans HA, Blankestijn PJ (2001) Cardiovascular risk factors in renal transplant patients: cyclosporin A versus tacrolimus. J Am Soc Nephrol 12: 368-373. [Crossref]

22. Wanner C, Bartens W (1994) Lipoprotein(a) in renal patients: is it a key factor in the high cardiovascular mortality? Nephrol Dial Transplant 9: 1066-1068.

23. Nemati E, Einollahi B, Taheri S, Moghani-Lankarani M, Kalantar E, et al. (2007) Cyclosporine trough $(\mathrm{C} 0)$ and 2-hour postdose $(\mathrm{C} 2)$ levels: which one is a predictor of graft loss? Transplant Proc 39: 1223-1224. [Crossref]

24. Ettinger WH, Bender WL, Goldberg AP, Hazzard WR (1987) Lipoprotein lipid abnormalities in healthy renal transplant recipients: persistence of low HDL2 cholesterol. Nephron 47: 17-21.

25. Ciftci HS, Ayna TK, Caliskan YK, Turkmen A, Gurtekin M (2013) Lipid parameters, doses and blood levels of calcineurin inhibitors in renal transplant patients. Indian $J$ Clin Biochem 28: 164-168.

26. Hager MR, Narla AD, Tannock LR (2017) Dyslipidemia in patients with chronic kidney disease. Rev Endocr Metab Disord 18: 29-40. [Crossref]

27. Molitch ME (2006) Management of dyslipidemias in patients with diabetes and chronic kidney disease. Clin J Am Soc Nephrol 1: 1090-1099. [Crossref]

28. Speer T, Zewinger S, Fliser D (2013) Uraemic dyslipidaemia revisited: role of highdensity lipoprotein. Nephrol Dial Transplant 28: 2456-2463.

29. Yau JC, Brunner LJ, Lopez-Berestein G, LeMaistre CF, Luke DR (1991) Therapeutic drug monitoring of cyclosporine-lipoprotein levels. Pharmacotherapy 11: 291-295.

Copyright: (2018 Laiq S. This is an open-access article distributed under the terms of the Creative Commons Attribution License, which permits unrestricted use, distribution, and reproduction in any medium, provided the original author and source are credited. 